\title{
Recent Developments in APT Analysis Automation and Support for User-Defined Custom Analysis Procedures in IVAS 4
}

\author{
A.C. Day ${ }^{1,2}$, H. Francois-Saint-Cyr ${ }^{2}$, B.P Geiser ${ }^{2}$, T. Payne ${ }^{2}$,E. Oltman ${ }^{2}$, S.P. Ringer ${ }^{1}$, D.A. Reinhard ${ }^{2}$ \\ 1. Australian Centre for Microscopy and Microanalysis, School of Aerospace, Mechanical and \\ Mechatronics Engineering, The University of Sydney, Sydney, NSW 2006, Australia. \\ 3. CAMECA Instruments Inc., 5470 Nobel Drive, Madison, WI 53711 USA. \\ * Corresponding author: alec.day@ametek.com
}

The Integrated Visualization and Analysis Software (IVAS ${ }^{\mathrm{TM}}$ ), developed by CAMECA Instruments Inc. offers a dedicated suite of tools to extract and analyze information from atom probe tomography (APT) data. IVAS also serves a critical purpose in the conversion of raw experimental file-types (RHIT and HITS), to user-readable file-types containing reconstructed APT data (POS and EPOS). It is this data that underpins and informs a very active area of research in the development of novel computational analysis procedures to extend the capabilities of APT [1-4] and enable new understandings in materials science $[5]$.

Traditionally, development of novel analysis procedures involves a complicated workflow between IVAS and various other programs and programming languages. Critical steps in the workflow path generally require some user input and the import/export of large data files, which often contains extraneous information redundant to the applied analysis procedure. These inefficiencies result in a bottleneck in the capacity to efficiently implement user-defined (third-party) developed codes and analysis procedures to APT data, particularly for large-scale studies across many datasets.

To improve data analysis workflow, we are introducing the custom analysis node in IVAS 4 (Figure 1). Here, custom analysis scripts for APT data can be imported, executed, visualized entirely within IVAS. This improves the productivity of applied analysis procedures by reducing the level of required user control and input along the workflow path. The IVAS interface is a command window with support for analysis scripts written in C\#. Other commonly used programming languages, such as Python and MATLAB $^{\circledR}$ can be executed through a prompt to the CMD terminal.

For codes written in C\#, the ion data can be directly read as an object. For other languages, the relevant information required by the custom script can be parsed through a new 'APT' file type. The APT file type is a flexible and extensible file type that supports storage of any type of required APT data and metadata including sparse data representation. As such, only the required information needs to be parsed into custom analysis scripts, decreasing the read-time and memory requirements over the traditional EPOS and POS file formats.

To enable the efficient application of both included and user-defined analysis procedures (constructed in the custom-analysis node) to multiple datasets, IVAS 4 introduces the automation flowchart. This provides the capability to construct and perform a complete workflow of repeatable analysis procedures from unreconstructed data through visualization and generation of output data, defined through an intuitive visual flow-chart. Although each analysis workflow can be entirely automated and applied to multiple datasets, some critical steps may benefit from some degree of user input. 
In this work, we present multiple studies based on pure Al samples, Al alloys containing Er precipitates, and finally steels containing $\mathrm{Cu}$ precipitates encompassing workflows that benefit from the custom analysis, and automation features implemented within IVAS 4. We focus on the reduction of workflow steps, to demonstrate the improvement of productivity and time-to-knowledge of applied analysis procedures. The studies are outlined as follows:

1. A systematic analysis of the effect of acquisition conditions in both straight flight-path and reflectron LEAP ${ }^{\circledR}$ instruments on the resolution of crystallographic planes in Al-based samples. Requiring a reconstruction protocol [1] and spatial distribution map analysis [4] performed with user-defined scripts executed within the custom analysis environment.

2. A workflow to apply user-defined clustering and precipitate analysis procedures [3] to multiple datasets reconstructed within IVAS.

User-defined scripts are coded in both Python and MATLAB to illustrate the flexibility of the custom analysis feature within IVAS 4.

\section{References:}

[1] A. Day, A. Ceguerra, S. Ringer, Microsc. Microanal. in press (2019), doi:10.1017/S1431927618015593

[2] Y. Wei et al., Ultramicroscopy 194 (2018), pp. 15-24.

[3] L. Stephenson et al., Microsc. Microanal. 13 (2007), pp. 448-463.

[4] N. Wallace et al., Ultramicroscopy 189 (2018), pp. 65-75.

[5] A. Devaraj et al., International Materials Reviews 63 (2018), pp. 68-101.

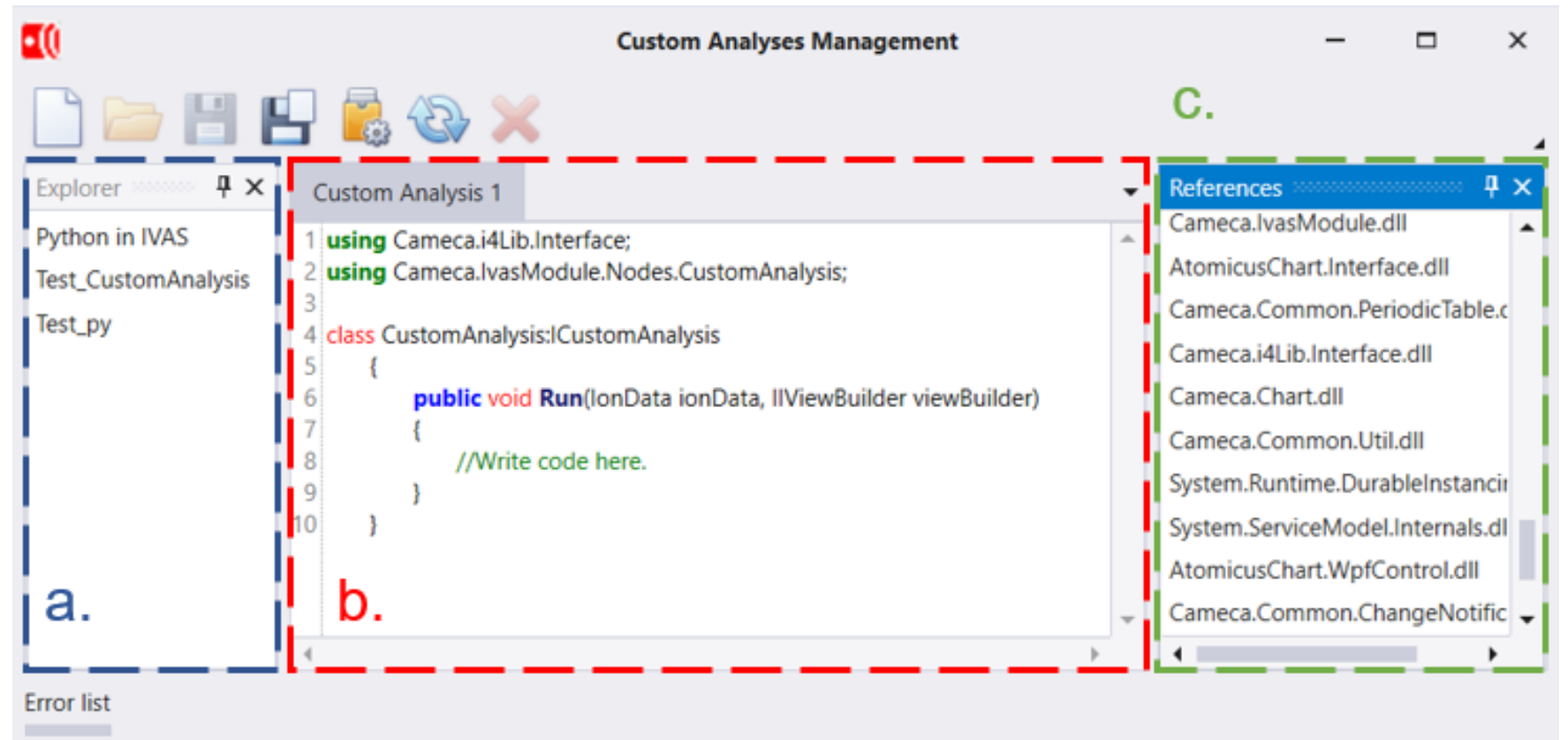

Figure 1. Draft specification for the custom analysis environment in IVAS 4. a. File explorer for saved custom analysis processes b. C\# command window to develop custom analysis scripts c. Inbuilt dependencies available to use in custom analysis, as .NET assemblies. 